\title{
AN ELEMENTARY NUMBER THEORETICAL REMARK
}

\section{E. HARZHEIM}

For a real number $r$ we define $((r))=r-[r]$, where $[r]$ is the greatest integer $\leqq r$. Then the number $e=\sum_{n=0}^{\infty} 1 / n$ ! has, as appears from this representation, the property that the values $((n ! \cdot e))$ converge to 0 monotonically, where specially $((n ! \cdot e))<1 / n$ holds $(n>0)$. This elementary fact can be imbedded into a general theorem on real numbers, because the following holds:

TheOREM. Let $r$ be a nonnegative real number. Then there exists a sequence $\alpha_{1}, \alpha_{2}, \cdots, \alpha_{n}, \cdots$, of nonnegative integers, such that the sequence

$$
\begin{aligned}
& a_{1}=\left(\left(r \cdot 1^{\alpha_{1}}\right)\right), \quad a_{2}=\left(\left(r \cdot 1^{\alpha_{1}} \cdot 2^{\alpha_{2}}\right)\right), \cdots, \\
& a_{n}=\left(\left(r \cdot 1^{\alpha_{1}} \cdot 2^{\alpha_{2}} \cdot 3^{\alpha_{3}} \cdots \cdot n^{\alpha_{n}}\right)\right), \cdots
\end{aligned}
$$

converges to 0 monotonically (in the wide sense), where especially the inequality $\left(\left(r \cdot 1^{\alpha_{1}} \cdot 2^{\alpha_{2}} \ldots \cdot n^{\alpha_{n}}\right)\right)<1 / n$ holds.

Proof. We put $\alpha_{1}=1$. If we have already defined $\alpha_{1}, \cdots, \alpha_{n}$, such that $a_{n}<1 / n$, it follows from this and from the formula

$$
\frac{1}{n}=\sum_{\nu=1}^{\infty} \frac{1}{(n-1)^{\nu}}
$$

either $a_{n}<1 /(n+1)$ or the existence of exactly one $k$ such that

$$
\begin{aligned}
& \frac{1}{n+1}+\frac{1}{(n+1)^{2}}+\cdots+\frac{1}{(n+1)^{k}} \\
& \quad \leqq a_{n}<\frac{1}{n+1}+\frac{1}{(n+1)^{2}}+\cdots+\frac{1}{(n+1)^{k}}+\frac{1}{(n+1)^{k+1}} .
\end{aligned}
$$

In the first case we put $\alpha_{n+1}=0$, in the second $\alpha_{n+1}=k$. Then it follows in both cases $a_{n+1}=\left(\left(a_{n} \cdot(n+1)^{\alpha_{n+1}}\right)\right)<1 /(n+1)$, such that the theorem is proved by induction.

If we associate in this way to every nonnegative real number $r$ the corresponding sequence $\alpha_{1}, \alpha_{2}, \cdots$, we can easily see, that $r$ is rational if and only if there exists a number $\nu$, such that $\alpha_{\mu}=0$ for all $\mu>\nu$. Now to $e$ there corresponds the sequence, where all $\alpha_{\nu}$ are equal 1.

Conversely it can be verified by the method of nested intervals

Received by the editors September 28, 1962. 
that to every sequence $\alpha_{1}, \alpha_{2}, \ldots$ (with $\alpha_{1}=1$ ) of nonnegative integers there exists exactly one real number in the interval $[0,1)$ to which the given sequence $\alpha_{1}, \alpha_{2}, \ldots$ corresponds.

Mathematisches Institut der Universität Köln, KöLn, Germany.

\section{A SHORT PROOF OF AN INEQUALITY FOR THE PERMANENT FUNCTION}

PETER M. GIBSON

Let $A$ be a substochastic matrix, i.e., a square matrix of nonnegative numbers with each row sum no greater than 1 . We have obtained a lower bound for the permanent of $I-A$.

Theorem. If $A$ is a substochastic matrix, then

$$
\text { per }(I-A) \geqq 0 \text {. }
$$

It was brought to our attention by Marcus and Minc [2] that Brualdi and Newman have proved this theorem. Indeed, two proofs of this theorem are contained in a paper that will appear in the Oxford Quarterly [1]. The proof that we shall give, shorter than and quite different from the Brualdi-Newman proofs, shows that this theorem is almost a corollary of the Ryser representation of the permanent.

Let $B$ be an $n$-square matrix and let $B_{r}$ denote a matrix obtained from $B$ by replacing some $r$ columns of $B$ by zero columns. Let $S\left(B_{r}\right)$ be the product of the row sums of the matrix $B_{r}$. Ryser [3] has proved that the permanent of $B$ is given by

$$
\begin{aligned}
\operatorname{per}(B)= & S\left(B_{0}\right)+\sum(-1) S\left(B_{1}\right)+\sum(-1)^{2} S\left(B_{2}\right)+\cdots \\
& +\sum(-1)^{n-1} S\left(B_{n-1}\right),
\end{aligned}
$$

where $\sum(-1)^{r} S\left(B_{r}\right)$ denotes the sum over all $\left(\begin{array}{l}n \\ r\end{array}\right)$ replacements of $r$ of the columns of $B$ by zero columns.

Let $B=I-A$ where $A$ is a substochastic matrix. The $i$ th row sum of $B_{r}$ is nonpositive or nonnegative according to whether the $i$ th column of $B_{r}$ is a zero or a nonzero column. Hence there are at least $r$ row sums of $B_{r}$ that are nonpositive and at least $n-r$ that are nonnegative. Therefore

Received by the editors September 2, 1965. 